\title{
Correlação entre a alteração da microbiota gastrintestinal com o surgimento e complicações na doença de Parkinson: papel de citocinas inflamatórias no eixo bidirecional intestino-cérebro
}

Correlation between changes in the gastrointestinal microbiota with the onset and complications of

Parkinson's disease: the role of inflammatory cytokines in the bidirectional gut-brain axis

Correlación entre los cambios en la microbiota gastrointestinal con la aparición y las

complicaciones de la enfermedad de Parkinson: el papel de las citocinas inflamatorias en el eje

bidireccional intestino-cerebro

Pablo Cleber Sousa Lopes Sales

ORCID: https://orcid.org/0000-0003-2617-9398 Faculdade de Ciências Humanas, Exatas e da Saúde do Piauí, IESVAP, Brasil E-mail: pablo.clebersales@gmail.com

Almir Vieira de Sousa Neto

ORCID: https://orcid.org/0000-0003-0620-3899

Faculdade de Ciências Humanas, Exatas e da Saúde do Piaú, IESVAP, Brasil E-mail: vieira10101almir@gmail.com

Lahuan Araujo Costa

ORCID: https://orcid.org/0000-0002-2490-2223

Faculdade de Ciências Humanas, Exatas e da Saúde do Piauí, IESVAP, Brasil

E-mail: Lahuan.araujo.costa@gmail.com

Mikhail de Morais Veras da Fonseca

ORCID: https://orcid.org/0000-0002-8667-2152

Faculdade de Ciências Humanas, Exatas e da Saúde do Piauí, IESVAP, Brasil E-mail: mikhailfonseca@gmail.com

Gabriela de Souza Mendonça

ORCID: https://orcid.org/0000-0002-7922-5995

Faculdade de Ciências Humanas, Exatas e da Saúde do Piauí, IESVAP, Brasil E-mail: gabyaraujo2007@ hotmail.com

Ligia Viana de Araújo

ORCID: https://orcid.org/0000-0002-0674-4604 Faculdade de Ciências Humanas, Exatas e da Saúde do Piauí, IESVAP, Brasil E-mail: ligiaviana00@gmail.com

Vitória Stefanny Cunha Araújo

ORCID: https://orcid.org/0000-0002-3901-2207

Faculdade de Ciências Humanas, Exatas e da Saúde do Piauí, IESVAP, Brasil

E-mail: Vitoriastefannycunhq@gmail.com

Ana Maria Santos Cardoso

ORCID: https://orcid.org/0000-0001-7092-2295

Faculdade de Ciências Humanas, Exatas e da Saúde do Piauí, IESVAP, Brasil

E-mail: anamariacardoso05@gmail.com

Paulo Victor de Sousa Jordão

ORCID: https://orcid.org/0000-0001-9227-6159

Faculdade de Ciências Humanas, Exatas e da Saúde do Piauí, IESVAP, Brasil E-mail: paulojordaopj@hotmail.com

Brenda Dias Araujo

ORCID: https://orcid.org/0000-0001-5140-0934

Faculdade de Ciências Humanas, Exatas e da Saúde do Piauí, IESVAP, Brasil E-mail: brenda150600@gmail.com

Charles Ponte de Sousa Filho

ORCID: https://orcid.org/0000-0002-0943-410X Faculdade de Ciências Humanas, Exatas e da Saúde do Piauí, IESVAP, Brasil E-mail: Charlesponte23@gmail.com

Louise Ribeiro Teixeira

ORCID: https://orcid.org/0000-0001-7378-1096

Faculdade de Ciências Humanas, Exatas e da Saúde do Piauí, IESVAP, Brasil

E-mail: ribeiroteixeiralouise@gmail.com 
José Guilherme de Oliveira Rodrigues Ferreira

ORCID: https://orcid.org/0000-0002-2617-5146

Faculdade de Ciências Humanas, Exatas e da Saúde do Piauí, IESVAP, Brasil

E-mail: jgorf13@gmail.com

Rafaela Costa Pacheco

ORCID: https://orcid.org/0000-0001-8477-8298

Faculdade de Ciências Humanas, Exatas e da Saúde do Piauí, IESVAP, Brasil

E-mail: pachecoc.rafaela@gmail.com

André Pessoa Silva Bastos

ORCID: https://orcid.org/0000-0002-1188-0766

Faculdade de Ciências Humanas, Exatas e da Saúde do Piauí, IESVAP, Brasil E-mail: Andrepsbastos@gmail.com

Brenda Ellen Menezes Cardoso

ORCID: https://orcid.org/0000-0002-5982-5985

Faculdade de Ciências Humanas, Exatas e da Saúde do Piauí, IESVAP, Brasil E-mail: cbrendaellen@ hotmail.com

Larruama Soares Figueiredo de Araújo

ORCID: https://orcid.org/0000-0002-0902-9200

Faculdade de Ciências Humanas, Exatas e da Saúde do Piauí, IESVAP, Brasil

E-mail: larru_pp@ hotmail.com

Bianca Sampaio Lima

ORCID: https://orcid.org/0000-0002-0574-4936

Faculdade de Ciências Humanas, Exatas e da Saúde do Piauí, IESVAP, Brasil E-mail: biancasp1234@gmail.com

Vivian Saeger Pires

ORCID: https://orcid.org/0000-0002-8852-0363

Faculdade de Ciências Humanas, Exatas e da Saúde do Piauí, IESVAP, Brasil E-mail: Viviansaegerpires@gmail.com

Luan Kelves Miranda de Souza

ORCID: https://orcid.org/0000-0002-8019-4022

Faculdade de Ciências Humanas, Exatas e da Saúde do Piauí, IESVAP, Brasil Universidade Federal do Piauí, Brasil E-mail: luan.souza@iesvap.edu.br

\begin{abstract}
Resumo
Os seres humanos desenvolveram uma relação simbiótica com sua microbiota intestinal, uma comunidade microbiana complexa composta de bactérias, archaea, protistas e vírus, incluindo bacteriófagos. O sistema nervoso entérico (SNE) é uma porta de entrada para a comunicação bidirecional entre o cérebro e o intestino, principalmente através do nervo vago (VN). Portanto, a exposição ambiental desempenha um papel fundamental tanto na composição quanto na funcionalidade do microbioma intestinal, e pode contribuir para a suscetibilidade a distúrbios neurodegenerativos, como a doença de Parkinson (DP). Trata-se de uma revisão sistemática de literatura onde foram utilizadas as bases de dados PUBMED, Scielo e MEDLINE, usando os descritores presentes nos Descritores em Ciências da Saúde (DeCS): doença de Parkinson, citocinas, microbiota e trato gastrintestinal. Pode-se mencionar que a característica neuropatológica da DP é a aparência generalizada de agregados de alfa-sinucleína ( $\alpha$-Syn) nos sistemas nervoso central e periférico, incluindo o SNE. Muitos estudos sugerem que as toxinas intestinais podem induzir a formação de agregados $\alpha$-Syn no SNE, que pode então ser transmitidos para o SNC através do VN. A DP está fortemente associada ao envelhecimento e seus efeitos negativos nos mecanismos homeostáticos que protegem da inflamação, do estresse oxidativo e do mau funcionamento proteico. Dessa forma, o referido estudo pretende realizar, a partir de análises das citocinas inflamatórias e da microbiota intestinal, como uma disbiose pode contribuir no surgimento ou complicações da DP, promovendo uma correlação de manifestações clínico-laboratoriais e prognósticos, bem como descrever os principais mecanismos causais que ainda são poucos compreendidos.
\end{abstract}

Palavras-chave: Microbiota; Doença de Parkinson; Neurodegeneração; Inflamação; Citocinas.

\begin{abstract}
Humans developed a symbiotic relationship with their gut microbiota, a complex microbial community made up of bacteria, archaea, protists and viruses, including bacteriophages. The enteric nervous system (SNE) is a gateway to bidirectional communication between the brain and the intestine, mainly through the vagus nerve (VN). Therefore, environmental exposure plays a fundamental role in both the composition and functionality of the intestinal microbiome and can contribute to susceptibility to neurodegenerative disorders, such as Parkinson's disease (PD). This is a systematic review of the literature using the data bases PUBMED, Scielo and MEDLINE, using the descriptors present in the Health Sciences Descriptors (DeCS): Parkinson's disease, cytokines, microbiota and gastrointestinal tract. It can be mentioned that the neuropathological characteristic of PD is the generalized appearance of alpha-synuclein ( $\alpha$-Syn) aggregates in the central and peripheral nervous systems, including the SNE. Many studies suggest that intestinal toxins can induce the formation of $\alpha$-Syn aggregates in the SNE, which can then be transmitted to the CNS via the VN. PD is strongly associated with aging and its negative effects on homeostatic mechanisms that protect against inflammation, oxidative stress and protein malfunction. Thus, this study intends to carry out, based on
\end{abstract}


analyzes of inflammatory cytokines and intestinal microbiota, how dysbiosis can contribute to the onset or complications of PD, promoting a correlation between clinical and laboratory manifestations and prognosis, as well as describing the main mechanisms causal factors that are still poorly understood.

Keywords: Microbiota; Parkinson's Disease; Neurodegeneration; Inflammation; Cytokines.

\begin{abstract}
Resumen
Los seres humanos han desarrollado una relación simbiótica con su microbiota intestinal, una comunidad microbiana compleja formada por bacterias, arqueas, protistas y virus, incluidos los bacteriófagos. El sistema nervioso entérico (SNE) es una puerta de entrada a la comunicación bidireccional entre el cerebro y el intestino, principalmente a través del nervio vago (VN). Por tanto, la exposición ambiental juega un papel fundamental tanto en la composición como en la funcionalidad del microbioma intestinal y puede contribuir a la susceptibilidad a los trastornos neurodegenerativos, como la enfermedad de Parkinson (EP). Se trata de una revisión sistemática de la literatura utilizando las bases de datos PUBMED, Scielo y MEDLINE, utilizando los descriptores presentes en los Descriptores de Ciencias de la Salud (DeCS): enfermedad de Parkinson, citocinas, microbiota y tracto gastrointestinal. Se puede mencionar que la característica neuropatológica de la EP es la aparición generalizada de agregados de alfa-sinucleína ( $\alpha$-Syn) en los sistemas nerviosos central y periférico, incluido el SNE. Muchos estudios sugieren que las toxinas intestinales pueden inducir la formación de agregados $\alpha$-Syn en el SNE, que luego pueden transmitirse al SNC a través de la VN. La EP está fuertemente asociada con el envejecimiento y sus efectos negativos sobre los mecanismos homeostáticos que protegen contra la inflamación, el estrés oxidativo y el mal funcionamiento de las proteínas. Así, este estudio pretende realizar, a partir de análisis de citocinas inflamatorias y microbiota intestinal, cómo la disbiosis puede contribuir a la aparición o complicaciones de la EP, promoviendo una correlación entre las manifestaciones clínicas y de laboratorio y el pronóstico, así como describir los principales mecanismos causales. factores que aún no se conocen bien.
\end{abstract}

Palabras clave: Microbiota; Enfermedad de Parkinson; Neurodegeneración; Inflamación; Citocinas.

\title{
1. Introdução
}

A doença de Parkinson (DP) é uma desordem neurodegenerativa progressiva crônica caracterizada pela morte precoce e proeminente de neurônios dopaminérgicos da parte compacta da substância negra (SNpc) e presença disseminada de alfasinucleína ( $\alpha$-Syn), uma proteína que atua a nível intracelular. Além disso, a deficiência de dopamina nos gânglios da base leva a sintomas motores parkinsonianos clássicos, como bradicinesia, tremor, rigidez e instabilidade postural posterior (Santos, et al.,2019).

Entretanto, acredita-se que a característica neuropatológica da DP seja promovida pela presença de inclusões citoplasmáticas, chamadas corpos de Lewy (LB) ou neurites de Lewy, em neurônios SNpc. Os LBs são compostos principalmente de agregados de $\alpha$-Syn, cujas conformações oligoméricas solúveis aberrantes são mediadas por seus efeitos tóxicos (Gründemann, et al., 2008).

A $\alpha$-Syn é uma proteína intrinsecamente desordenada (IDP), que não possui estrutura 3D estável sob condições fisiológicas e é caracterizada por plasticidade estrutural exacerbada e adaptabilidade conformacional. Dessa forma, assim como outras IDPs, que possuem regiões amiloidogênicas, $\alpha$-Syn pode se transformar em um ligante promíscuo levando a interações anormais e ao desenvolvimento de DP (Stefanis, 2012).

A DP também está associada a sintomas não motores, que podem preceder os sintomas motores em mais de uma década como, por exemplo, disfunção olfatória e gastrointestinal. Nesse sentido, foi demonstrado cientificamente, que agregados de fibrilas de $\alpha$-Syn também são encontrados em tecidos localizados fora do sistema nervoso central (SNC) de pacientes com DP como, por exemplo, no sistema nervoso autônomo e também no sistema nervoso entérico (SNE) (Radhakrishnan e Goyal, 2018).

Dessa forma, pode-se inferir que existe uma intensa comunicação bidirecional entre o SNC e SNE através do eixo intestino-cérebro e, que, a partir do momento que existir alguma disfunção ao longo dessa base comunicacional pode levar o organismo a apresentar severos problemas, dentre eles a própria DP. Ainda é possível mencionar que esse diálogo orquestrado acontece por meio de conexões recíprocas que são mediadas pelo nervo pélvico e, principalmente, pelo nervo vago (NV). Portanto, o NV acaba sendo um bom alvo para o uso de terapias de neuroestimulação para o tratamento de distúrbios 
psiquiátricos e gastrointestinais (Miraglia e Colla, 2019).

Nesse contexto, é importante salientar que o trato gastrointestinal abriga um ecossistema microbiano complexo, consistindo de bactérias, archaea, protistas, vírus, dentre outros (Strandwitz, et al., 2019; Gogokhia et al., 2019; Darby et al., 2019). Dessa forma, pode-se mencionar que o microbioma humano evoluiu em conjunto com o hospedeiro e mantém um controle rígido sobre a natureza competitiva intrínseca dos microrganismos que compõem sua microbiota, através dos sistemas nervoso e imunológico. Esse arranjo maximiza os benefícios que o hospedeiro ganha com a relação simbiótica, incluindo proteção contra patógenos, melhoria da nutrição e saúde mental (Santos, et al.; 2019; Miraglia e Colla, 2019).

Existem evidências crescentes da associação entre a disfunção do microbioma e as comorbidades relacionadas ao SNC, como ansiedade, depressão, transtornos do espectro do autismo, doença de Alzheimer e doença de Parkinson. Essa associação provavelmente surgiu como um subproduto das forças de seleção natural que agem nos microrganismos para se adaptar ao hospedeiro e vice-versa (Gogokhia, et al., 2019).

O efeito da microbiota no SNC pode levar a modificações de comportamento e até mesmo à manipulação do hospedeiro associado ao aumento da aptidão de suas populações bacterianas. Cita-se, por exemplo, que a microbiota pode influenciar nas interações sociais, agindo sobre o comportamento nutricional de animais, particularmente aqueles de espécies sociais, onde os indivíduos compartilham microrganismos e interagem em torno dos alimentos. Além disso, os mecanismos neuroendocrinológicos e inflamatórios adjacentes a esse tipo de manipulação são amplamente compartilhados pelo hospedeiro através do seu microbioma (Darby, et al., 2019).

Portanto, menciona-se, por exemplo, os níveis de muitos neurotransmissores que são importantes para a expressão do comportamento social bem como para a saúde mental dos indivíduos. Vale a pena ressaltar ainda que, o processo inflamatório iniciado por uma disbiose a nível gastrintestinal pode provocar, de forma inicial, os principais sinais e sintomas associados a fase não motora da doença de Parkinson (Miraglia \& Colla, 2019).

Esses fatores causam temeridade e acionam um estado de alerta para que novos alvos sejam estudados, uma vez que a incidência e prevalência da DP aumenta com o avanço da idade, estando presente em 1\% das pessoas com mais de 65 anos, além de acometer faixas etárias cada vez mais jovens como é o caso da DP de início precoce e da DP "juvenil" (Radhakrishnan e Goyal, 2018).

Atualmente, a base do manejo da DP é a terapia farmacológica; entretanto, essas terapias sintomáticas têm grandes limitações na doença avançada. Muitas características incapacitantes se desenvolvem mais tarde no curso da doença, incluindo sintomas não motores, sintomas motores resistentes à dopamina e complicações motoras da terapia com dopamina a longo prazo. Ainda se pode mencionar que boa parte da intervenção farmacológica não leva em consideração o aspecto microbiano instalado no indivíduo e tão pouco remetem ao processo inflamatório causado por um desarranjo desses microrganismos a nível gastrointestinal (Hasegawa, et al., 2015).

Posto isso, esse estudo possui caráter fulcral, uma vez que objetiva esclarecer algumas lacunas que não são bem descritas na DP, além de compreender como um processo inflamatório a nível gastrointestinal, promovido por alteração da microbiota, pode afetar diretamente o eixo bidirecional intestino-cérebro, dando ênfase no papel de citocinas inflamatórias ao longo desse eixo.

\section{Metodologia}

De acordo com Pereira. et al. (2018), esse estudo trata-se de uma revisão sistemática da literatura, com abordagem qualitativa, haja vista a interpretação e análise dos elementos obtidos. A pergunta norteadora do estudo foi: “Qual a relação entre a DP e o eixo bidirecional intestino-cérebro?”, seguindo posteriormente as etapas de: identificação do tema, seleção da hipótese ou questão de pesquisa, estabelecimento de critérios de inclusão e exclusão, definição das informações a serem 
extraídas, avaliação dos estudos incluídos na revisão, interpretação dos resultados e apresentação da revisão com síntese do conhecimento. A hipótese estabelecida foi: “O eixo gut-brain, em sinergia com a disbiose e as citocinas inflamatórias, está envolvido diretamente na etiologia da DP”. Os critérios de inclusão definidos para a seleção dos artigos foram: artigos que estivessem disponíveis na íntegra com acesso on-line nas bases de dados pesquisadas, publicados no período entre os anos de 2008 a 2021. Possuiu como critérios de exclusão o período, uma vez que não foram selecionados artigos anteriores a 2008, e literatura cinzenta, ou seja, material de literatura não controlada por editores científicos ou comerciais.

Foram utilizadas as seguintes bases de dados: Biblioteca Nacional de Medicina dos Estados Unidos (PUBMED), Scientific Eletronic Library Online (Scielo), Medical Literature Analysis and Retrieval System on-line (MEDLINE), usando os descritores presentes nos Descritores em Ciências da Saúde (DeCS) que são: doença de Parkinson, citocinas, microbiota e trato gastrintestinal. Esses descritores foram combinados de dois em dois com o intuito de encontrar o maior número possível de publicações adequadas para a revisão. Para análise dos artigos, foi realizada leitura na íntegra dos estudos. Após essa etapa, foi realizada discussão entre os autores, solucionando as divergências por meio da releitura dessas publicações, por conseguinte, foram selecionados quinze artigos. Para melhor compreensão, os métodos serão demonstrados na Figura 1.

Figura 1 - Fluxograma esquematizando a metodologia do estudo.

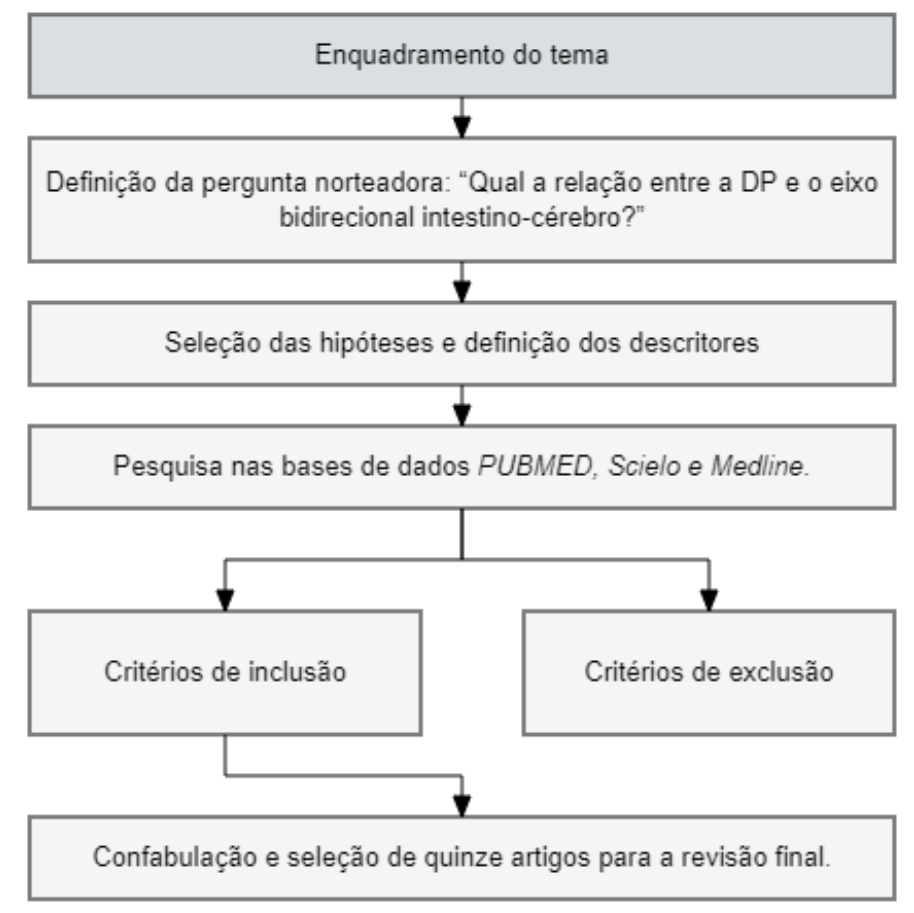

Fonte: Autores.

O esquema mostrado acima tem a função de apresentar de forma rápida e eficiente a metodologia do projeto, destacando as etapas separadamente e mostrando por um esquema de vetores (de cima para baixo) a evolução e sequência dessas etapas.

\section{Resultados e Discussão}

O parkinsonismo pode ser definido como uma grande categoria de doenças que podem apresentar redução na neurotransmissão dopaminérgica nos gânglios da base, estando estas classificadas em: parkinsonismo primário, secundário, plus e heredodegenerativas. Cabe salientar também que esse tipo de patologia vem se fazendo mais presente na população 
mundial, principalmente em indivíduos em uma faixa etária mais avançada (Souza, et al., 2011).

Sabe-se que a DP afeta cerca de 1 a 2 pessoas 1000 da população, sendo que sua prevalência aumenta gradativamente com a idade, afetando cerca $1 \%$ da população acima de 60 anos. O principal achado neuropatológico da DP são corpos de Lewy, que contém agregados de $\alpha$-sinucleína - $\alpha$-Syn, uma proteína intracelular. A causa da DP é desconhecida na maioria dos casos, mas há uma forte relação de fatores genéticos, incluindo causas monogênicas, sendo que algum fator genético pode ser identificado em 5-10\% dos pacientes. Ademais, outros vários fatores ambientais estão associados ao aumento do risco de DP (Kim, et al, 2017; Tysnes, 2017).

Essa desordem neurodegenerativa progressiva crônica é caracterizada pela morte precoce proeminente de neurônios dopaminérgicos na pars compacta substantia nigra (SNpc) e presença disseminada de $\alpha$-Syn. Dessa maneira, a deficiência de dopamina nos gânglios da base leva a sintomas motores parkinsonianos clássicos, como bradicinesia, tremor, rigidez e instabilidade postural posterior (Stefanis, et al, 2012).

A DP também está associada a sintomas não motores, que podem preceder os sintomas motores em mais de uma década. Esses sintomas não motores tornam-se sintomas incômodos nos últimos estágios da DP. Atualmente, a base do manejo da DP é a terapia farmacológica; entretanto, essas terapias sintomáticas têm grandes limitações na doença avançada. Muitas características incapacitantes se desenvolvem mais tarde no curso da doença, incluindo sintomas não motores, sintomas motores resistentes à dopamina e complicações motoras da terapia com dopamina a longo prazo (Radhakrishnan e Goyal, 2018).

Embora tenha havido avanços notáveis no tratamento médico e cirúrgico da DP, a terapia definitiva de modificação da doença não existe. No entanto, novas pesquisas têm sido elaboradas para que possa ser possível a identificação de novos alvos potenciais para a modificação da doença. Sob essa óptica, sabe-se que existe uma possibilidade de que uma disbiose da microbiota presente no indivíduo possa ser um dos iniciadores da DP bem como de suas complicações, principalmente pelo o surgimento de processos inflamatórios (Gazerani, 2019; Darby, et al., 2019).

Nesse contexto, pode-se mencionar que os nervos parassimpáticos e o sistema nervoso entérico estão entre as estruturas mais afetadas pela patologia de promovida pela grande deposição de $\alpha$-Syn. A associação do sistema nervoso ao sistema gastrointestinal, através do eixo bidirecional intestino cérebro, mediado pelo nervo vago (VN-via) e pelas vias humorais, como o tecido linfático e a corrente sanguínea (Via não-VN) se mostra como um fator importante no estudo dos fatores de risco da DP e que merece cada vez mais enfoque da comunidade científica (Miraglia e Colla, 2019). Com efeito, podem existir disfunções ao longo do eixo microbiota-intestino-cérebro que podem estar associadas a sintomas não motores presentes na DP, apoiando assim a hipótese de que o processo patológico se espalha em um eixo bidirecional partindo da região intestinal para a cerebral. Portanto, a microbiota intestinal pode desempenhar um importante papel na regulação dos distúrbios do movimento e as alterações na microbiota podem ser importante fator de risco para a DP (Hasegawa, et al., 2015).

Além disso, é importante salientar que uma monocamada de células epiteliais separa o lúmen intestinal e o complexo microbioma intestinal dos tecidos nervosos linfóides e entéricos subjacentes (Gazerani, 2019; Santos, et al., 2019). Nesse contexto ainda é possivel mencionar que componentes do microbioma intestinal e seus compostos extracelulares podem desencadear respostas no VN através de células enteroendócrinas, que são contatadas pelos terminais do nervo vago através de estruturas especializadas chamadas neuropods (NP) (Radhakrishnan e Goyal, 2018).

Com isso, antígenos microbianos podem atravessar o epitélio intestinal através de células especializadas, que podem desempenhar um papel central em respostas inflamatórias localizadas, e ainda é possível inferir a participação de Receptores toll-like nesse processo. Esses receptores são proteínas sensíveis a microrganismos, presentes nas células epiteliais do intestino, mediando o reconhecimento de bactérias comensais das bactérias nocivas/ inflamatórias (Strandwitz, et al., 2019).

Prova disso são alguns estudos que relacionam a incidência de DP a doenças inflamatórias intestinais (DII), como a Doença de Crohn e a retocolite ulcerativa, sendo que até o momento, cinco coortes de base populacional relataram que o risco 
de DP é aumentado em pacientes com DII, com um risco entre 20\% 90\% maior de desenvolver DP do que indivíduos sem DII (Lee, Lobbestael e Vermiere 2021). No entanto, a incidência de DII entre pacientes com DP continua sendo um tópico de intensa discussão, visto que outro estudo mostrou que os pacientes com DP tiveram 15\% menor risco de desenvolver DC e RU do que os controles não-DP (Camacho-Soto, Gross, Nielsen, Dey \& Racette, 2018).

Sincrônico, citocinas foram diretamente implicados na patogênese das DII em estudos genéticos e imunológicos recentes, e parecem ter um papel crucial no controle inflamatório. Estudos de DII em modelos de camundongos mostraram que a modulação da função da citocina pode, inclusive, ser usada para terapia, além disso, o papel principal delas também tem relevância devido ao fato de que o bloqueio do fator de necrose tumoral (TNF) também vem sendo estudado como alternativa de tratamento às DII e, possivelmente, uma futura terapia preventiva à DP (Neurath, 2014). Infere-se, portanto, que apesar do risco de DP entre os pacientes com DII ser elevado, o inverso não parece seguir a mesma tendência.

Dessa forma, é notório que algumas evidências mostram que a disbiose associada ao intestino e os componentes microbianos, gerando processos inflamatórios, são importantes agentes e fatores de risco para a DP. Portanto, o microbioma intestinal surge como um alvo potencial para medidas de proteção com o objetivo de prevenir o aparecimento da doença de Parkinson (Gogokhia, et al, 2019).

\section{Considerações Finais}

A DP é uma doença neurodegenerativa na qual múltiplos fatores podem estar associados à sua etiologia, nesse sentido, a saúde do intestino se mostra como um possível agente no desenvolvimento da DP, tendo a microbiota intestinal um papel fundamental nisso, haja vista que estudos evidenciam a relação entre a disbiose associada ao intestino e os componentes da microbiota intestinal gerando processos inflamatórios, que, consequentemente, são fatores de risco para essa doença.

Por conseguinte, a proteção do microbioma intestinal surge como uma alternativa potencial para o desenvolvimento de medidas de proteção e prevenção à DP. Dessa forma, espera-se que a partir dessa pesquisa haja, em trabalhos futuros, o aumento do conhecimento sobre os fatores de risco dessa doença, como a participação do eixo bidirecional intestino-cérebro, de modo a aprimorar as formas de diagnóstico e manejo da DP e minimizar os efeitos colaterais oriundos do tratamento tradicional, melhorando assim a qualidade de vida dos indivíduos acometidos e reduzindo os gastos do Sistema Público de Saúde em assistência à saúde.

\section{Referências}

Camacho-Soto, A., Gross, A., Nielsen, S. S., Dey, N., \& Racette, B. A. (2018). Inflammatory bowel disease and risk of Parkinson's disease in Medicare beneficiaries. Parkinsonism \& related disorders, 50, 23-28.

Darby, T. M., Owens, J. A., Saeedi, B. J., Luo, L., Matthews, J. D., Robinson, B. S., \& Jones, R. M. (2019). Lactococcus Lactis Subsp. cremoris is an efficacious beneficial bacterium that limits tissue injury in the intestine. Iscience, 12, 356-367.

Gazerani, P. (2019). Probiotics for Parkinson's disease. International journal of molecular sciences, 20(17), 4121.

Gogokhia, L., Buhrke, K., Bell, R., Hoffman, B., Brown, D. G., Hanke-Gogokhia, C., \& Round, J. L. (2019). Expansion of bacteriophages is linked to aggravated intestinal inflammation and colitis. Cell host \& microbe, 25(2), 285-299.

Gründemann, J., Schlaudraff, F., Haeckel, O., \& Liss, B. (2008). Elevated $\alpha$-synuclein mRNA levels in individual UV-laser-microdissected dopaminergic substantia nigra neurons in idiopathic Parkinson's disease. Nucleic acids research, 36(7), e38.

Hasegawa, S., Goto, S., Tsuji, H., Okuno, T., Asahara, T., Nomoto, K., \& Hirayama, M. (2015). Intestinal dysbiosis and lowered serum lipopolysaccharidebinding protein in Parkinson's disease. PloS one, 10(11), e0142164.

Kim, D. S., Choi, H. I., Wang, Y., Luo, Y., Hoffer, B. J., \& Greig, N. H. (2017). A new treatment strategy for Parkinson's disease through the gut-brain axis: the glucagon-like peptide-1 receptor pathway. Cell transplantation, 26(9), 1560-1571.

Lee, H. S., Lobbestael, E., Vermeire, S., Sabino, J., \& Cleynen, I. (2021). Inflammatory bowel disease and Parkinson's disease: common pathophysiological links. Gut, 70(2), 408-417.

Miraglia, F., \& Colla, E. (2019). Microbiome, Parkinson's disease and molecular mimicry. Cells, 8(3), 222. 
Research, Society and Development, v. 10, n. 2, e51310212788, 2021

(CC BY 4.0) | ISSN 2525-3409 | DOI: http://dx.doi.org/10.33448/rsd-v10i2.12788

Neurath, M. F. (2014). Cytokines in inflammatory bowel disease. Nature Reviews Immunology, 14(5), 329-342.

Pereira, A. S., Shitsuka, D. M., Parreira, F. J., \& Shitsuka, R. (2018). Metodologia da pesquisa científica.

Radhakrishnan, D. M., \& Goyal, V. (2018). Parkinson's disease: A review. Neurology India, 66(7), 26.

Santos, S. F., De Oliveira, H. L., Yamada, E. S., Neves, B. C., \& Pereira Jr, A. (2019). The gut and Parkinson's Disease, a bidirectional pathway. Frontiers in neurology, 10, 574 .

Souza, C. F. M., Almeida, H. C. P., Sousa, J. B., Costa, P. H., Silveira, Y. S. S., \& Bezerra, J. C. L. (2011). A doença de parkinson e o processo de envelhecimento motor. Revista Neurociências, 19(4), 718-723.

Stefanis, L. (2012). $\alpha$-Synuclein in Parkinson's disease. Cold Spring Harbor perspectives in medicine, $2(2)$, a009399.

Strandwitz, P., Kim, K. H., Terekhova, D., Liu, J. K., Sharma, A., Levering, J., \& Lewis, K. (2019). GABA-modulating bacteria of the human gut microbiota. Nature microbiology, 4(3), 396-403.

Tysnes, O. B., \& Storstein A. Epidemiology of Parkinson's disease (2017). J Neural Transm. 124 (8), 901-905. 